CRYSTALLOGRAPHIC COMMUNICATIONS

ISSN 2056-9890

Received 7 April 2020

Accepted 30 April 2020

Edited by A. J. Lough, University of Toronto, Canada

Keywords: crystal structure; $\mathrm{C}-\mathrm{H} \cdots \pi$ hydrogen bond; Hirschfeld surface analysis; adamantane; terminal alkyne.

CCDC reference: 2000259

Supporting information: this article has supporting information at journals.iucr.org/e

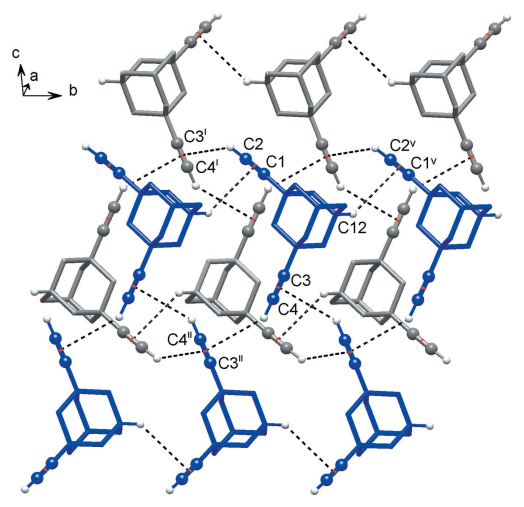

OPEN $\odot$ ACCESS

\section{Crystal structure and Hirshfeld surface analysis of 1,3-diethynyladamantane}

\author{
Kostiantyn V. Domasevitch* and Anna S. Degtyarenko
}

Inorganic Chemistry Department, National Taras Shevchenko University of Kyiv, Volodymyrska Str. 64/13, 01601 Kyiv, Ukraine. *Correspondence e-mail: dk@univ.kiev.ua

The title compound, $\mathrm{C}_{14} \mathrm{H}_{16}$, exhibits exceptionally weak intermolecular $\mathrm{C}-$ $\mathrm{H} \cdots \pi$ hydrogen bonding of the ethynyl groups, with the corresponding $\mathrm{H} \cdots \pi$ separations [2.91 (2) and 3.12 (2) $\AA$ ] exceeding normal vdW distances. This bonding complements distal contacts of the $\mathrm{CH}$ (aliphatic) $\cdots \pi$ type $[\mathrm{H} \cdots \pi=$ $3.12(2)-3.14$ (2) $\AA$ ] to sustain supramolecular layers. Hirshfeld surface analysis of the title compound suggests a relatively limited significance of the $\mathrm{C} \cdots \mathrm{H} /$ $\mathrm{H}$.. C contacts to the crystal packing (24.6\%) and a major contribution from $\mathrm{H} \cdots \mathrm{H}$ contacts accounting $74.9 \%$ to the entire surface.

\section{Chemical context}

Terminal alkynes provide self-complementary hydrogen-bond donor and acceptor functionality to sustain weak $\mathrm{C}-\mathrm{H} \cdots \pi$ interactions (Nishio, 2004). The latter dominate the crystal structure of acetylene (McMullan et al., 1992). In the case of polyfunctional species, the significance of such $\mathrm{C}-\mathrm{H} \cdots \pi$ interactions is rather low, since only $13.3 \%$ of related structures exhibit this kind of bonding (Allen et al., 2013). This may be associated with the specific geometry demands that concern an orthogonal orientation of the donor and acceptor alkyne groups. It is not surprising that examples for $\mathrm{C}-\mathrm{H} \cdots \pi$-driven self-assembly of terminal diynes are particularly rare. These examples are restricted to a few structures of hydrocarbons lacking stronger supramolecular interactions. Most of the literature precedents, such as 1,4-diethynylbenzene (Weiss et al., 1997), 1,4-diethynylcubane (Eaton et al., 1994) and $\alpha, \omega$ octa- and decadiynes (Bond, 2002) feature collinear orientations of the ethynyl groups within the molecules, which are beneficial for the generation of the simplest of supramolecular patterns. In the case of angular diynes, the demands of dense molecular packing may be less compatible with highly directional orthogonal interactions of $\mathrm{C} \equiv \mathrm{CH}$ (donor) and $\mathrm{C} \equiv \mathrm{CH}$ (acceptor) groups. One can anticipate the essential distortion and weakening (if not elimination at all) of the $\mathrm{C}-\mathrm{H} \cdots \pi$ bonding.

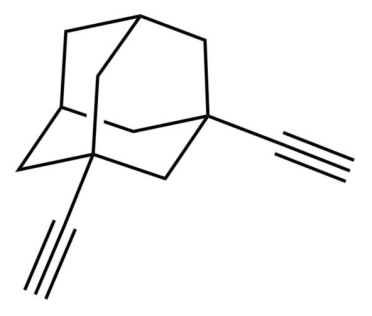

In this context, we have examined the angular compound 1,3-diethynyladamantane and report its crystal structure 
herein. The crystal packing of 1,3-disubstituted adamantanes also recently attracted attention in the context of polymorphism and the formation of plastic phases (Negrier et al., 2020).

\section{Structural commentary}

The molecular structure of the title compound is shown in Fig. 1. The bonds lengths in the carbocyclic framework

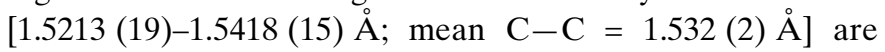
typical for adamantane derivatives, for example 1,3-diphenyladamantane with mean $\mathrm{C}-\mathrm{C}=1.530$ (6) $\AA$ (Tukada \& Mochizuki, 2003). At the same time, these bonds are slightly shorter than those observed for an adamantane-1,3-diyl core bearing two electron-donor groups, such as 1,3-dimethyl[mean $\mathrm{C}-\mathrm{C}=1.562(6) \AA]$ and 1,3-dihydroxyadamantanes [mean $\mathrm{C}-\mathrm{C}=1.563$ (2) $\AA$ ] (Negrier et al., 2020). The alkyne fragments $\mathrm{C} 5-\mathrm{C} 1 \equiv \mathrm{C} 2$ and $\mathrm{C} 7-\mathrm{C} 3 \equiv \mathrm{C} 4$ are linear, with the corresponding bond angles being 177.47 (13) and 178.31 (12) ${ }^{\circ}$, respectively. The geometries of these fragments $[\mathrm{C} 1 \equiv \mathrm{C} 2=$ $1.1763(17) ; \quad \mathrm{C} 3 \equiv \mathrm{C} 4=1.1812(19) \AA$ and $\mathrm{C} 1-\mathrm{C} 5=$ $1.4708(15), \mathrm{C} 3-\mathrm{C} 7=1.4673(16) \AA]$ are consistent with the data for non-conjugated terminal alkynes, for example 1,7octadiyne [1.186 (2) and 1.464 (2) A, respectively; Bond, 2002].

\section{Supramolecular features}

Hydrogen-bond interactions of the alkyne groups are exceptionally weak and there are no $\mathrm{H} \cdots \pi$ separations ( $\pi$ is defined as a centroid of the triple-bonded atoms) falling into the interval of 2.39-2.90 ̊ suggested by Allen et al. (2013). Even the shortest related contact $\left[\mathrm{C} 1 \mathrm{C} 2 \mathrm{H} \cdots \mathrm{C} 4^{\mathrm{i}}=2.905\right.$ (18) $\AA$; symmetry code: (i) $x,-\frac{1}{2}-y, \frac{1}{2}+z$ ], is longer than the normal vdW separation of $2.87 \AA$ (Zefirov, 1997). In particular, the

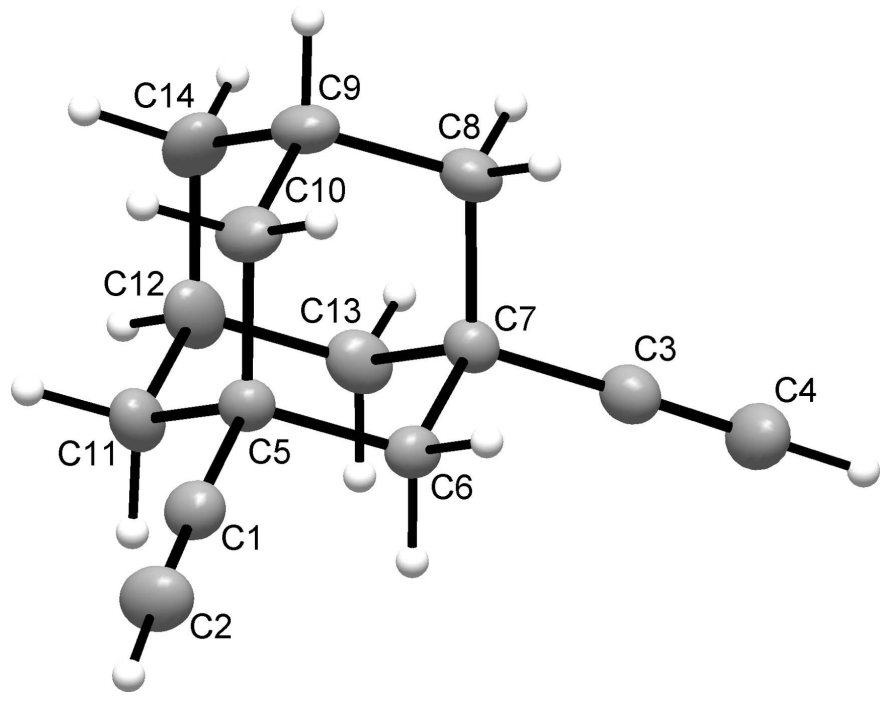

Figure 1

The molecular structure of the title compound, showing the atom-labeling scheme. Displacement ellipsoids are drawn at the $40 \%$ probability level and the $\mathrm{H}$ atoms are shown as small spheres of arbitrary radii.
Table 1

Geometry of the shortest $\mathrm{C}-\mathrm{H} \cdots \pi$ contacts $\left(\AA{ }^{\circ}{ }^{\circ}\right)$.

$\mathrm{Cg}$ is a group centroid.

\begin{tabular}{lcccc}
\hline$D-\mathrm{H} \cdots \pi$ & $D-\mathrm{H}$ & $\mathrm{H} \cdots \pi$ & $D \cdots A$ & $D-\mathrm{H} \cdots \pi$ \\
\hline \multicolumn{5}{l}{ Contacts with ethyne CH donors } \\
$\mathrm{C} 2-\mathrm{H} 2 \cdots C g(\mathrm{C} 3 \mathrm{C} 4)^{\mathrm{i}}$ & $0.927(19)$ & $2.91(2)$ & $3.679(2)$ & $140.7(14)$ \\
$\mathrm{C} 4-\mathrm{H} 4 \cdots C g(\mathrm{C} 3 \mathrm{C} 4)^{\mathrm{ii}}$ & $0.96(2)$ & $3.12(2)$ & $3.958(2)$ & $146.5(14)$ \\
\multicolumn{5}{l}{ Contacts with aliphatic CH donors } \\
$\mathrm{C} 6-\mathrm{H} 6 B \cdots C g(\mathrm{C} 3 \mathrm{C} 4)^{\mathrm{iii}}$ & $0.970(13)$ & $3.12(2)$ & $4.030(2)$ & $155.9(10)$ \\
$\mathrm{C} 10-\mathrm{H} 10 A \cdots C g(\mathrm{C} 1 \mathrm{C} 2)^{\mathrm{iv}}$ & $0.957(16)$ & $3.14(2)$ & $3.853(2)$ & $133.0(10)$ \\
$\mathrm{C} 12-\mathrm{H} 12 \cdots C g(\mathrm{C} 1 \mathrm{C} 2)^{\mathrm{v}}$ & $0.967(16)$ & $3.14(2)$ & $3.904(2)$ & $136.7(12)$ \\
\hline
\end{tabular}

Symmetry codes: (i) $x,-\frac{1}{2}-y, \frac{1}{2}+z$; (ii) $-x,-\frac{1}{2}+y,-\frac{1}{2}-z$; (iii) $-x,-y,-z$; (iv) $1-x$, $\frac{1}{2}+y, \frac{1}{2}-z ;$ (v) $x, 1+y, z$.

distal interactions of the $\mathrm{C} 3 \equiv \mathrm{C} 4 \mathrm{H}$ donors $[\mathrm{H} \cdots \pi=$ 3.12 (2) $\AA$ ] do not differ in geometry from a set of $\mathrm{H} \cdots \pi$ contacts established by the methylene (C6 and C10) and methyne (C12) groups (Table 1). Both ethynyl groups are donors of such $\mathrm{CH} \cdots \pi$ bonding, whereas their acceptor functions are not uniform. The $\mathrm{C} 3 \equiv \mathrm{C} 4 \mathrm{H}$ groups accept two $\mathrm{C} \equiv \mathrm{CH} \cdots \pi$ bonds and establish an additional comparable contact with an aliphatic donor, while the $\mathrm{C} 1 \equiv \mathrm{C} 2 \mathrm{H}$ groups maintain only two distal contacts with the aliphatic $\mathrm{CH}$ portion. Mutual bonding of $\mathrm{C} 3 \equiv \mathrm{C} 4 \mathrm{H}$ groups $[\mathrm{H} \cdots \pi=$ 3.12 (2) $\AA$; symmetry code: (ii) $-x,-\frac{1}{2}+y,-\frac{1}{2}-z$ ] as well as contacts with the methyne groups $\mathrm{C} 12 \mathrm{H} \cdots \mathrm{Cg}(\mathrm{C} 1 \mathrm{C} 2)^{\mathrm{v}}[\mathrm{H} \cdots \pi$ $=3.14$ (2) $\AA$; $C g$ is a group centroid; symmetry code: (v) $x, 1+y$, $z$ ] link the molecules into zigzag chains along the $b$-axis direction (Fig. 2). These aggregate into layers, which are

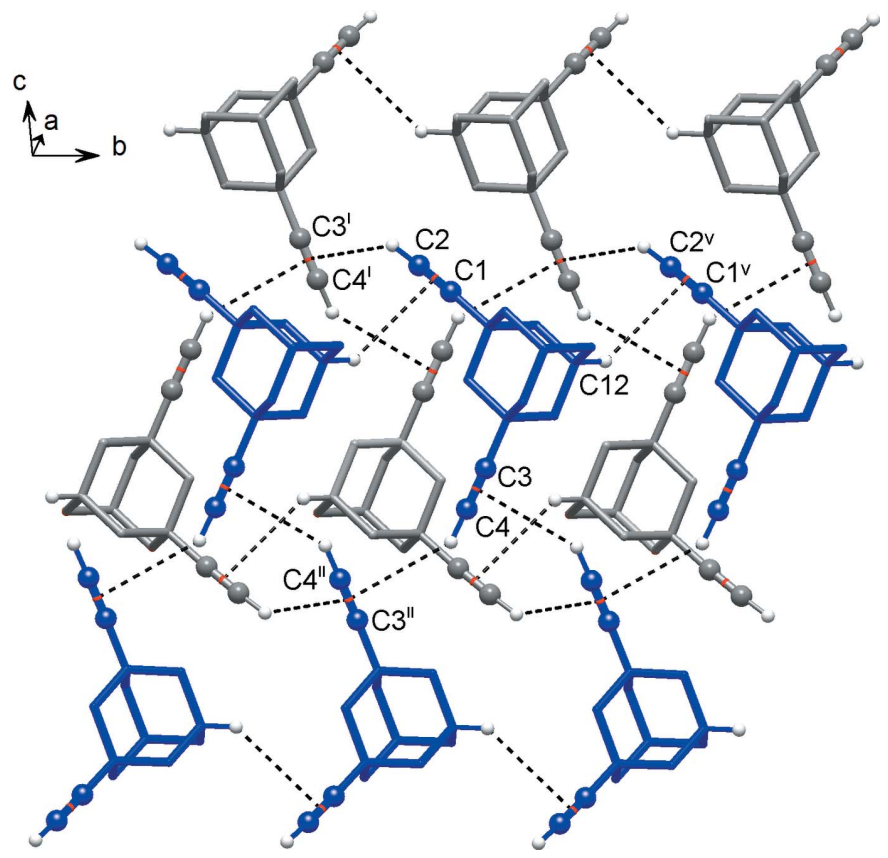

Figure 2

Fragment of the title crystal structure showing two zigzag chains (marked in blue and grey) running along the $b$-axis direction in the crystal, with a set of shortest $\mathrm{C}-\mathrm{H} \cdots \pi$ contacts indicated by dashed lines [symmetry codes: (i) $x,-\frac{1}{2}-y, \frac{1}{2}+z$; (ii) $-x,-\frac{1}{2}+y,-\frac{1}{2}-z$; (v) $\left.x, 1+y, z\right]$. 


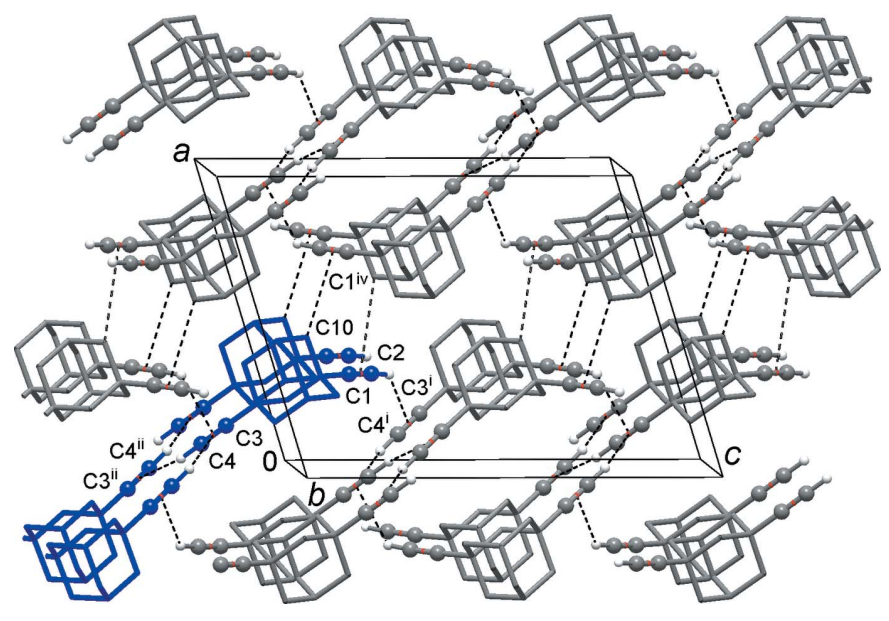

Figure 3

Packing of the $\mathrm{C}-\mathrm{H} \cdots \pi$-bonded chains with the formation of layers, which are parallel to the $b c$ plane. The blue color identifies a single chain that is marked in a similar manner in Fig. 2, and dashed lines indicate C$\mathrm{H} \cdots \pi$ contacts within the layer and methylene $\cdots \pi$ contacts between adjacent layers. [Symmetry codes: (i) $x,-\frac{1}{2}-y, \frac{1}{2}+z$; (ii) $-x,-\frac{1}{2}+y$, $-0.5-z$; (iv) $1-x, \frac{1}{2}+y, \frac{1}{2}-z$.]

parallel to the $b c$ plane with a set of the above bonds involving $\mathrm{C} 1 \equiv \mathrm{C} 2 \mathrm{H}$ donors and $\mathrm{C} 3 \equiv \mathrm{C} 4 \mathrm{H}\left(x,-\frac{1}{2}-y, \frac{1}{2}+z\right)$ acceptors. The shortest contacts between successive layers concern interactions involving the methylene groups $\mathrm{C} 10 \mathrm{H} \cdots \mathrm{Cg}(\mathrm{C} 1 \mathrm{C} 2)^{\mathrm{iv}}[\mathrm{H} \cdots \pi=3.14$ (2) $\AA$ A ; symmetry code: (iv) $1-x, \frac{1}{2}+y, \frac{1}{2}-z$; Fig. 3].

The $\mathrm{C} \equiv \mathrm{CH} \cdots \pi$ geometries reported here are only approximately comparable with the parameters of much stronger and more directional supramolecular bonding in 1,4diethynylbenzene $\left[\mathrm{H} \cdots \pi=2.72 \AA\right.$; $\left.\mathrm{C}-\mathrm{H} \cdots \pi=175^{\circ}\right]$ (Weiss et al., 1997). More important is that even very weak and bifurcated $\mathrm{C}-\mathrm{H} \cdots \pi$ bonds in $\alpha, \omega$-octa- and decadiynes $[\mathrm{H} \cdots \pi=2.99-3.03 \AA$; Bond, 2002] are superior to those reported here based upon single and well-defined acceptors. The weakness of the $\mathrm{C} \equiv \mathrm{CH} \cdots \pi$ bonds in the title structure and their limited significance are best illustrated by their peer interplay and competition with aliphatic $\mathrm{C}-\mathrm{H} \cdots \pi$ contacts, with the corresponding interatomic separations exceeding the sum of vdW radii.

\section{Hirshfeld analysis}

The supramolecular interactions in the title structure have been further investigated and visualized by Hirshfeld surface analysis (Spackman \& Byrom, 1997; McKinnon et al., 2004; Hirshfeld, 1977) performed with CrystalExplorer17 (Turner et al., 2017). The Hirshfeld surface of the molecule, mapped over $d_{\text {norm }}$ in the color range 0.0957 to 1.3378 a.u., indicates only a set of normal vdW contacts (white regions) corresponding to the closest interactions (Fig. 4). The two-dimensional fingerprint plot is appreciably reminiscent of the one for adamantane itself (Spackman \& McKinnon, 2002), but accompanied by two additional diffuse features appearing as wings at the top left and bottom right of the plot (Fig. 5). These wings

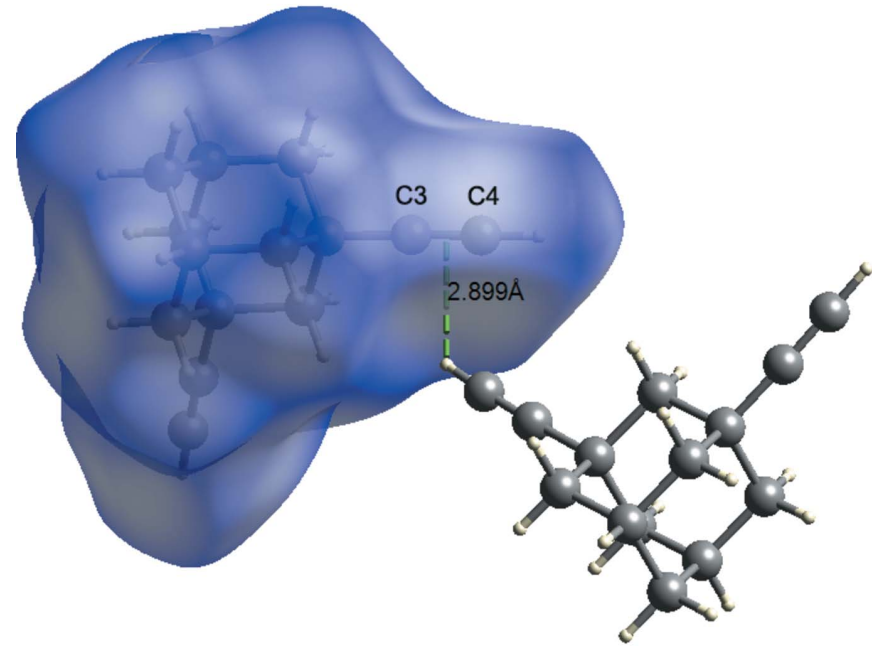

Figure 4

The Hirshfeld surface of the title compound mapped over $d_{\text {norm }}$ in the color range 0.0957 to 1.3378 a.u. showing the shortest $\mathrm{H} \cdots \pi$ contact with the normalized $\mathrm{C}-\mathrm{H}$ distance.

correspond to a series of $\mathrm{C} \cdot \mathrm{H} / \mathrm{H} \cdots \mathrm{C}$ contacts. Nevertheless, $\mathrm{H} \cdot \mathrm{H}$ contacts (the shortest ones are at the $d_{\mathrm{e}}=d_{\mathrm{i}}=1.2 \AA$ level) are by far the major contributors $(74.9 \%)$ to the entire surface, while the fraction of $\mathrm{C} \cdot \mathrm{H} / \mathrm{H} \cdots \mathrm{C}$ contacts accounts for only $24.6 \%$. The latter value may be compared with contributions of 40.0 and $32.4 \%$ calculated for $\alpha, \omega$-octa- and decadiynes (Bond, 2002) and this significant suppression of the $\mathrm{C} \cdot \mathrm{H} / \mathrm{H} \cdots \mathrm{C}$ contacts is in line with the very weak $\mathrm{C}-\mathrm{H} \cdots \pi$ bonding in the title structure, as described above. There are no stacking interactions of the ethynyl groups: the contribution of the $\mathrm{C} \cdots \mathrm{C}$ contacts to the entire surface does not exceed $0.5 \%$.
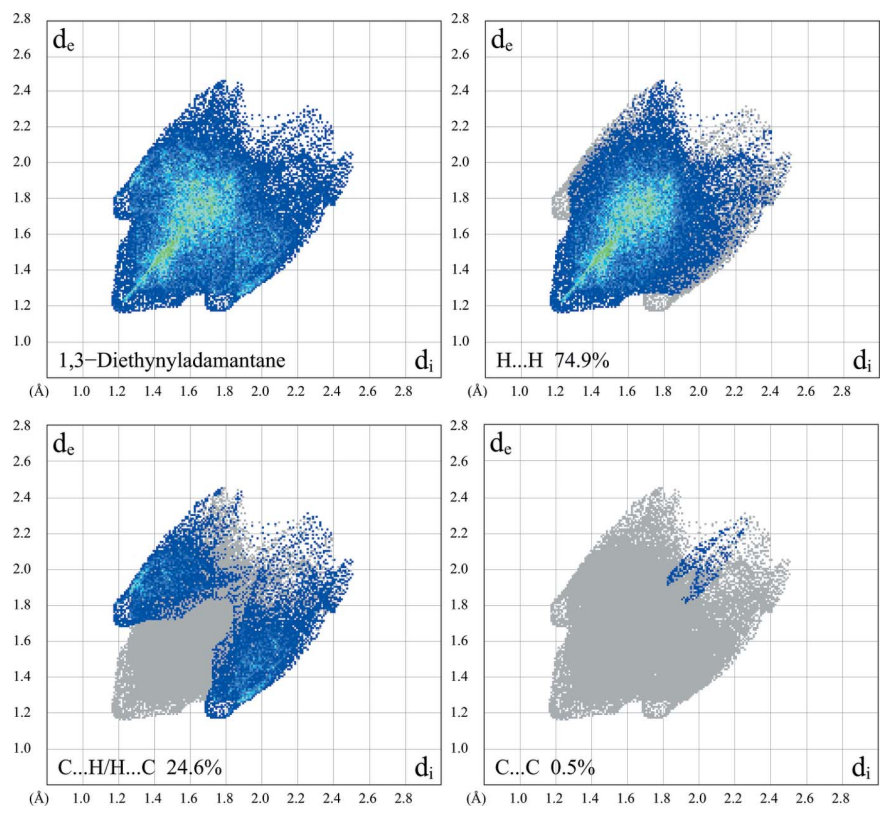

Figure 5

The two-dimensional fingerprint plot for the title compound, and those delineated into $\mathrm{H} \cdots \mathrm{H}(74.9 \%), \mathrm{C} \cdots \mathrm{H} / \mathrm{H} \cdots \mathrm{C}(24.6 \%)$ and $\mathrm{C} \cdots \mathrm{C}(0.5 \%)$ contacts. 
Table 2

Experimental details.

\begin{tabular}{|c|c|}
\hline \multicolumn{2}{|l|}{ Crystal data } \\
\hline Chemical formula & $\mathrm{C}_{14} \mathrm{H}_{16}$ \\
\hline$M_{\mathrm{r}}$ & 184.27 \\
\hline Crystal system, space group & Monoclinic, $P 2_{1} / c$ \\
\hline Temperature (K) & 213 \\
\hline$a, b, c(\AA)$ & $\begin{array}{l}11.3214(9), 6.7426(6) \\
14.9478(12)\end{array}$ \\
\hline$\beta\left({ }^{\circ}\right)$ & $107.234(9)$ \\
\hline$V\left(\AA^{6}\right)$ & $1089.82(16)$ \\
\hline$Z$ & 4 \\
\hline Radiation type & Мo $K \alpha$ \\
\hline$\mu\left(\mathrm{mm}^{-1}\right)$ & 0.06 \\
\hline Crystal size $(\mathrm{mm})$ & $0.26 \times 0.23 \times 0.20$ \\
\hline \multicolumn{2}{|l|}{ Data collection } \\
\hline Diffractometer & Stoe IPDS \\
\hline $\begin{array}{l}\text { No. of measured, independent and } \\
\text { observed }[I>2 \sigma(I)] \text { reflections }\end{array}$ & $9458,2593,1885$ \\
\hline$R_{\text {int }}$ & 0.039 \\
\hline$(\sin \theta / \lambda)_{\max }\left(\AA^{-1}\right)$ & 0.661 \\
\hline \multicolumn{2}{|l|}{ Refinement } \\
\hline$R\left[F^{2}>2 \sigma\left(F^{2}\right)\right], w R\left(F^{2}\right), S$ & $0.044,0.124,0.99$ \\
\hline No. of reflections & 2593 \\
\hline No. of parameters & 191 \\
\hline $\mathrm{H}$-atom treatment & All $\mathrm{H}$-atom parameters refined \\
\hline$\Delta \rho_{\max }, \Delta \rho_{\min }\left(\mathrm{e} \AA^{-3}\right)$ & $0.29,-0.17$ \\
\hline
\end{tabular}

Computer programs: IPDS Software (Stoe \& Cie, 2000), SHELXS97 (Sheldrick, 2008), SHELXL2018/1 (Sheldrick, 2015), DIAMOND (Brandenburg, 1999) and WinGX (Farrugia, 2012).

\section{Synthesis and crystallization}

The title compound was synthesized in a three-step reaction sequence starting with selective dibromination of adamantane (Degtyarenko et al., 2014). The reaction product was crystallized from methanol.

\section{Refinement}

Crystal data, data collection and structure refinement details are summarized in Table 2. The non-H atoms were refined with anisotropic displacement parameters. All hydrogen atoms were located in a difference maps and then freely refined with isotropic displacement parameters $[\mathrm{C}-\mathrm{H}$ (ethynyl) $=0.927(19)$ and $0.96(2) \AA ; \mathrm{C}-\mathrm{H}$ (methyne) = 0.967 (16) and 0.971 (16) $\AA$; C-H (methylene) $=0.952$ (14)1.013 (19) ^̊].

\section{Funding information}

This work was supported by the Ministry of Education and Science of Ukraine (project No. 19BF037-05).

\section{References}

Allen, F. H., Wood, P. A. \& Galek, P. T. A. (2013). Acta Cryst. B69, 281-287.

Bond, A. D. (2002). Chem. Commun. pp. 1664-1665.

Brandenburg, K. (1999). DIAMOND. Crystal Impact GbR, Bonn, Germany.

Degtyarenko, A. S., Handke, M., Krämer, K. W., Liu, S.-X., Decurtins, S., Rusanov, E. B., Thompson, L. K., Krautscheid, H. \& Domasevitch, K. V. (2014). Dalton Trans. 43, 8530-8542.

Eaton, P. E., Galoppini, E. \& Gilardi, R. (1994). J. Am. Chem. Soc. 116, 7588-7596.

Farrugia, L. J. (2012). J. Appl. Cryst. 45, 849-854.

Hirshfeld, F. L. (1977). Theor. Chim. Acta, 44, 129-138.

McKinnon, J. J., Spackman, M. A. \& Mitchell, A. S. (2004). Acta Cryst. B60, 627-668.

McMullan, R. K., Kvick, Å. \& Popelier, P. (1992). Acta Cryst. B48, 726-731.

Negrier, P., Ben Hassine, B., Barrio, M., Romanini, M., Mondieig, D. \& Tamarit, J.-L. (2020). CrystEngComm, 22, 1230-1238.

Nishio, M. (2004). CrystEngComm, 6, 130-158.

Sheldrick, G. M. (2008). Acta Cryst. A64, 112-122.

Sheldrick, G. M. (2015). Acta Cryst. C71, 3-8.

Spackman, M. A. \& Byrom, P. G. A. (1997). Chem. Phys. Lett. 267, 215-220.

Spackman, M. A. \& McKinnon, J. J. (2002). CrystEngComm, 4, 378392.

Stoe \& Cie (2000). IPDS Software. Stoe \& Cie GmbH, Darmstadt, Germany.

Tukada, H. \& Mochizuki, K. (2003). J. Mol. Struct. 655, 473-478.

Weiss, H.-C., Bläser, D., Boese, R., Doughan, B. M. \& Haley, M. M. (1997). Chem. Commun. pp. 1703-1704.

Zefirov, Y. V. (1997). Crystallogr. Rep. 42, 865-886. 


\section{supporting information}

Acta Cryst. (2020). E76, 807-810 [https://doi.org/10.1107/S2056989020005964]

\section{Crystal structure and Hirshfeld surface analysis of 1,3-diethynyladamantane}

\section{Kostiantyn V. Domasevitch and Anna S. Degtyarenko}

\section{Computing details}

Data collection: IPDS Software (Stoe \& Cie, 2000); cell refinement: IPDS Software (Stoe \& Cie, 2000); data reduction: IPDS Software (Stoe \& Cie, 2000); program(s) used to solve structure: SHELXS97 (Sheldrick, 2008); program(s) used to refine structure: SHELXL2018/1 (Sheldrick, 2015); molecular graphics: DIAMOND (Brandenburg, 1999); software used to prepare material for publication: WinGX (Farrugia, 2012).

(I)

Crystal data

$\mathrm{C}_{14} \mathrm{H}_{16}$

$M_{r}=184.27$

Monoclinic, $P 2{ }_{1} / c$

$a=11.3214$ (9) $\AA$

$b=6.7426(6) \AA$

$c=14.9478(12) \AA$

$\beta=107.234(9)^{\circ}$

$V=1089.82(16) \AA^{3}$

$Z=4$

$F(000)=400$

$D_{\mathrm{x}}=1.123 \mathrm{Mg} \mathrm{m}^{-3}$

Mo $K \alpha$ radiation, $\lambda=0.71073 \AA$

Cell parameters from 8000 reflections

$\theta=3.3-28.0^{\circ}$

$\mu=0.06 \mathrm{~mm}^{-1}$

$T=213 \mathrm{~K}$

Prism, colorless

$0.26 \times 0.23 \times 0.20 \mathrm{~mm}$

Data collection

Stoe IPDS

diffractometer

Radiation source: fine-focus sealed tube

$\varphi$ oscillation scans

9458 measured reflections

2593 independent reflections

\section{Refinement}

Refinement on $F^{2}$

Least-squares matrix: full

$R\left[F^{2}>2 \sigma\left(F^{2}\right)\right]=0.044$

$w R\left(F^{2}\right)=0.124$

$S=0.99$

2593 reflections

191 parameters

0 restraints

Primary atom site location: structure-invariant direct methods
1885 reflections with $I>2 \sigma(I)$

$R_{\text {int }}=0.039$

$\theta_{\max }=28.0^{\circ}, \theta_{\min }=3.3^{\circ}$

$h=-14 \rightarrow 14$

$k=-8 \rightarrow 8$

$l=-19 \rightarrow 19$

Secondary atom site location: difference Fourier map

Hydrogen site location: difference Fourier map

All $\mathrm{H}$-atom parameters refined

$w=1 /\left[\sigma^{2}\left(F_{\mathrm{o}}^{2}\right)+(0.086 P)^{2}\right]$

where $P=\left(F_{\mathrm{o}}^{2}+2 F_{\mathrm{c}}^{2}\right) / 3$

$(\Delta / \sigma)_{\max }<0.001$

$\Delta \rho_{\max }=0.29 \mathrm{e} \AA^{-3}$

$\Delta \rho_{\min }=-0.17 \mathrm{e} \AA^{-3}$ 


\section{Special details}

Geometry. All esds (except the esd in the dihedral angle between two 1.s. planes) are estimated using the full covariance matrix. The cell esds are taken into account individually in the estimation of esds in distances, angles and torsion angles; correlations between esds in cell parameters are only used when they are defined by crystal symmetry. An approximate (isotropic) treatment of cell esds is used for estimating esds involving l.s. planes.

Fractional atomic coordinates and isotropic or equivalent isotropic displacement parameters $\left(\AA^{2}\right)$

\begin{tabular}{lllll}
\hline & $x$ & $y$ & $z$ & $U_{\text {iso }} / U_{\text {eq }}$ \\
\hline C1 & $0.28206(10)$ & $-0.04337(17)$ & $0.21845(8)$ & $0.0389(3)$ \\
C2 & $0.27667(12)$ & $-0.1572(2)$ & $0.27676(9)$ & $0.0504(3)$ \\
C3 & $0.11946(10)$ & $0.07256(18)$ & $-0.11933(8)$ & $0.0398(3)$ \\
C4 & $0.05502(13)$ & $0.0009(2)$ & $-0.18880(10)$ & $0.0543(4)$ \\
C5 & $0.28328(9)$ & $0.09619(15)$ & $0.14311(7)$ & $0.0322(2)$ \\
C6 & $0.20113(9)$ & $0.01414(14)$ & $0.04951(7)$ & $0.0303(2)$ \\
C7 & $0.19844(9)$ & $0.15694(15)$ & $-0.03144(7)$ & $0.0314(2)$ \\
C8 & $0.33158(10)$ & $0.18505(18)$ & $-0.03602(9)$ & $0.0396(3)$ \\
C9 & $0.41216(11)$ & $0.2689(2)$ & $0.05677(9)$ & $0.0456(3)$ \\
C10 & $0.41510(10)$ & $0.1250(2)$ & $0.13649(9)$ & $0.0435(3)$ \\
C11 & $0.23145(13)$ & $0.29881(17)$ & $0.16114(9)$ & $0.0421(3)$ \\
C12 & $0.22953(13)$ & $0.44094(16)$ & $0.08120(9)$ & $0.0457(3)$ \\
C13 & $0.14768(11)$ & $0.35843(16)$ & $-0.01119(9)$ & $0.0406(3)$ \\
C14 & $0.36049(14)$ & $0.46899(19)$ & $0.07563(11)$ & $0.0548(4)$ \\
H2 & $0.2661(16)$ & $-0.248(3)$ & $0.3202(13)$ & $0.077(5)^{*}$ \\
H4 & $0.0043(17)$ & $-0.063(3)$ & $-0.2445(14)$ & $0.072(5)^{*}$ \\
H6A & $0.2336(11)$ & $-0.1137(19)$ & $0.0358(9)$ & $0.036(3)^{*}$ \\
H6B & $0.1179(12)$ & $-0.0042(17)$ & $0.0536(9)$ & $0.036(3)^{*}$ \\
H8A & $0.3640(14)$ & $0.055(2)$ & $-0.0489(10)$ & $0.049(4)^{*}$ \\
H8B & $0.3328(12)$ & $0.269(2)$ & $-0.0870(10)$ & $0.042(3)^{*}$ \\
H9 & $0.4956(14)$ & $0.283(2)$ & $0.0525(11)$ & $0.057(4)^{*}$ \\
H10A & $0.4668(14)$ & $0.175(2)$ & $0.1950(11)$ & $0.053(4)^{*}$ \\
H10B & $0.4484(14)$ & $-0.008(2)$ & $0.1243(11)$ & $0.052(4)^{*}$ \\
H11A & $0.1490(15)$ & $0.280(2)$ & $0.1688(11)$ & $0.058(4)^{*}$ \\
H11B & $0.2825(14)$ & $0.356(2)$ & $0.2211(11)$ & $0.052(4)^{*}$ \\
H12 & $0.1974(14)$ & $0.567(2)$ & $0.0945(11)$ & $0.056(4)^{*}$ \\
H13A & $0.1440(14)$ & $0.445(2)$ & $-0.0645(11)$ & $0.055(4)^{*}$ \\
H13B & $0.0615(15)$ & $0.340(2)$ & $-0.0080(11)$ & $0.055(4)^{*}$ \\
H14A & $0.3613(15)$ & $0.561(2)$ & $0.0255(12)$ & $0.061(4)^{*}$ \\
H14B & $0.4157(17)$ & $0.527(3)$ & $0.1360(14)$ & $0.074(5)^{*}$ \\
& & & &
\end{tabular}

Atomic displacement parameters $\left(\AA^{2}\right)$

\begin{tabular}{lllllll}
\hline & $U^{11}$ & $U^{22}$ & $U^{33}$ & $U^{12}$ & $U^{13}$ & $U^{23}$ \\
\hline C1 & $0.0379(5)$ & $0.0402(6)$ & $0.0369(6)$ & $-0.0020(4)$ & $0.0086(5)$ & $0.0026(5)$ \\
C2 & $0.0523(7)$ & $0.0520(7)$ & $0.0450(7)$ & $-0.0029(6)$ & $0.0112(6)$ & $0.0153(6)$ \\
C3 & $0.0396(6)$ & $0.0448(6)$ & $0.0369(6)$ & $0.0016(5)$ & $0.0142(5)$ & $0.0037(5)$ \\
C4 & $0.0516(7)$ & $0.0702(9)$ & $0.0391(7)$ & $-0.0116(6)$ & $0.0102(6)$ & $-0.0021(6)$ \\
C5 & $0.0346(5)$ & $0.0304(5)$ & $0.0328(6)$ & $-0.0021(4)$ & $0.0120(4)$ & $0.0029(4)$
\end{tabular}




\begin{tabular}{lllllll} 
C6 & $0.0315(5)$ & $0.0256(5)$ & $0.0353(6)$ & $0.0008(4)$ & $0.0122(4)$ & $0.0014(4)$ \\
C7 & $0.0329(5)$ & $0.0306(5)$ & $0.0327(5)$ & $0.0022(4)$ & $0.0127(4)$ & $0.0018(4)$ \\
C8 & $0.0381(6)$ & $0.0458(6)$ & $0.0404(7)$ & $-0.0007(5)$ & $0.0202(5)$ & $0.0029(5)$ \\
C9 & $0.0362(6)$ & $0.0577(7)$ & $0.0459(7)$ & $-0.0133(5)$ & $0.0166(5)$ & $0.0038(6)$ \\
C10 & $0.0323(5)$ & $0.0549(7)$ & $0.0413(7)$ & $-0.0056(5)$ & $0.0079(5)$ & $0.0046(5)$ \\
C11 & $0.0579(7)$ & $0.0335(6)$ & $0.0404(7)$ & $-0.0029(5)$ & $0.0232(6)$ & $-0.0043(5)$ \\
C12 & $0.0689(8)$ & $0.0238(5)$ & $0.0505(7)$ & $0.0003(5)$ & $0.0271(6)$ & $-0.0015(5)$ \\
C13 & $0.0486(6)$ & $0.0308(5)$ & $0.0461(7)$ & $0.0097(5)$ & $0.0197(5)$ & $0.0088(5)$ \\
C14 & $0.0748(9)$ & $0.0412(7)$ & $0.0505(8)$ & $-0.0249(6)$ & $0.0218(7)$ & $-0.0010(6)$ \\
\hline
\end{tabular}

Geometric parameters $\left(\AA,{ }^{o}\right)$

\begin{tabular}{|c|c|c|c|}
\hline $\mathrm{C} 1-\mathrm{C} 2$ & $1.1763(17)$ & $\mathrm{C} 8-\mathrm{H} 8 \mathrm{~B}$ & $0.952(14)$ \\
\hline $\mathrm{C} 1-\mathrm{C} 5$ & $1.4708(15)$ & $\mathrm{C} 9-\mathrm{C} 10$ & $1.5293(18)$ \\
\hline $\mathrm{C} 2-\mathrm{H} 2$ & $0.927(19)$ & $\mathrm{C} 9-\mathrm{C} 14$ & $1.530(2)$ \\
\hline $\mathrm{C} 3-\mathrm{C} 4$ & $1.1812(19)$ & $\mathrm{C} 9-\mathrm{H} 9$ & $0.971(16)$ \\
\hline $\mathrm{C} 3-\mathrm{C} 7$ & $1.4673(16)$ & $\mathrm{C} 10-\mathrm{H} 10 \mathrm{~A}$ & $0.957(16)$ \\
\hline $\mathrm{C} 4-\mathrm{H} 4$ & $0.96(2)$ & $\mathrm{C} 10-\mathrm{H} 10 \mathrm{~B}$ & $1.012(15)$ \\
\hline $\mathrm{C} 5-\mathrm{C} 6$ & $1.5354(15)$ & $\mathrm{C} 11-\mathrm{C} 12$ & $1.5269(16)$ \\
\hline $\mathrm{C} 5-\mathrm{C} 10$ & $1.5370(14)$ & $\mathrm{C} 11-\mathrm{H} 11 \mathrm{~A}$ & $0.982(15)$ \\
\hline $\mathrm{C} 5-\mathrm{C} 11$ & $1.5418(15)$ & C11-H11B & $0.988(16)$ \\
\hline $\mathrm{C} 6-\mathrm{C} 7$ & $1.5396(14)$ & $\mathrm{C} 12-\mathrm{C} 14$ & $1.5213(19)$ \\
\hline C6-H6A & $0.982(13)$ & $\mathrm{C} 12-\mathrm{C} 13$ & $1.5222(19)$ \\
\hline C6-H6B & $0.970(13)$ & $\mathrm{C} 12-\mathrm{H} 12$ & $0.967(16)$ \\
\hline $\mathrm{C} 7-\mathrm{C} 13$ & $1.5396(14)$ & $\mathrm{C} 13-\mathrm{H} 13 \mathrm{~A}$ & $0.980(16)$ \\
\hline $\mathrm{C} 7-\mathrm{C} 8$ & $1.5408(13)$ & C13-H13B & $0.999(16)$ \\
\hline $\mathrm{C} 8-\mathrm{C} 9$ & $1.5250(18)$ & $\mathrm{C} 14-\mathrm{H} 14 \mathrm{~A}$ & $0.976(17)$ \\
\hline $\mathrm{C} 8-\mathrm{H} 8 \mathrm{~A}$ & $0.993(14)$ & C14-H14B & $1.013(19)$ \\
\hline $\mathrm{C} 2-\mathrm{C} 1-\mathrm{C} 5$ & $177.47(13)$ & $\mathrm{C} 10-\mathrm{C} 9-\mathrm{H} 9$ & $108.6(9)$ \\
\hline $\mathrm{C} 1-\mathrm{C} 2-\mathrm{H} 2$ & $175.6(11)$ & $\mathrm{C} 14-\mathrm{C} 9-\mathrm{H} 9$ & $110.9(9)$ \\
\hline $\mathrm{C} 4-\mathrm{C} 3-\mathrm{C} 7$ & $178.31(12)$ & $\mathrm{C} 9-\mathrm{C} 10-\mathrm{C} 5$ & $109.45(10)$ \\
\hline $\mathrm{C} 3-\mathrm{C} 4-\mathrm{H} 4$ & $177.5(11)$ & $\mathrm{C} 9-\mathrm{C} 10-\mathrm{H} 10 \mathrm{~A}$ & $110.9(9)$ \\
\hline $\mathrm{C} 1-\mathrm{C} 5-\mathrm{C} 6$ & $109.07(8)$ & $\mathrm{C} 5-\mathrm{C} 10-\mathrm{H} 10 \mathrm{~A}$ & $109.2(8)$ \\
\hline $\mathrm{C} 1-\mathrm{C} 5-\mathrm{C} 10$ & $111.08(9)$ & $\mathrm{C} 9-\mathrm{C} 10-\mathrm{H} 10 \mathrm{~B}$ & $110.3(9)$ \\
\hline $\mathrm{C} 6-\mathrm{C} 5-\mathrm{C} 10$ & $108.93(9)$ & $\mathrm{C} 5-\mathrm{C} 10-\mathrm{H} 10 \mathrm{~B}$ & $108.7(8)$ \\
\hline $\mathrm{C} 1-\mathrm{C} 5-\mathrm{C} 11$ & $110.04(9)$ & $\mathrm{H} 10 \mathrm{~A}-\mathrm{C} 10-\mathrm{H} 10 \mathrm{~B}$ & $108.3(12)$ \\
\hline $\mathrm{C} 6-\mathrm{C} 5-\mathrm{C} 11$ & $108.63(9)$ & $\mathrm{C} 12-\mathrm{C} 11-\mathrm{C} 5$ & $109.71(9)$ \\
\hline $\mathrm{C} 10-\mathrm{C} 5-\mathrm{C} 11$ & $109.04(9)$ & $\mathrm{C} 12-\mathrm{C} 11-\mathrm{H} 11 \mathrm{~A}$ & $112.4(9)$ \\
\hline $\mathrm{C} 5-\mathrm{C} 6-\mathrm{C} 7$ & $110.88(8)$ & $\mathrm{C} 5-\mathrm{C} 11-\mathrm{H} 11 \mathrm{~A}$ & $109.1(9)$ \\
\hline $\mathrm{C} 5-\mathrm{C} 6-\mathrm{H} 6 \mathrm{~A}$ & $110.0(7)$ & $\mathrm{C} 12-\mathrm{C} 11-\mathrm{H} 11 \mathrm{~B}$ & $109.6(8)$ \\
\hline $\mathrm{C} 7-\mathrm{C} 6-\mathrm{H} 6 \mathrm{~A}$ & $107.8(7)$ & $\mathrm{C} 5-\mathrm{C} 11-\mathrm{H} 11 \mathrm{~B}$ & $110.6(9)$ \\
\hline $\mathrm{C} 5-\mathrm{C} 6-\mathrm{H} 6 \mathrm{~B}$ & $109.0(7)$ & $\mathrm{H} 11 \mathrm{~A}-\mathrm{C} 11-\mathrm{H} 11 \mathrm{~B}$ & $105.4(12)$ \\
\hline $\mathrm{C} 7-\mathrm{C} 6-\mathrm{H} 6 \mathrm{~B}$ & $109.6(7)$ & $\mathrm{C} 14-\mathrm{C} 12-\mathrm{C} 13$ & $109.69(10)$ \\
\hline $\mathrm{H} 6 \mathrm{~A}-\mathrm{C} 6-\mathrm{H} 6 \mathrm{~B}$ & $109.4(10)$ & $\mathrm{C} 14-\mathrm{C} 12-\mathrm{C} 11$ & $109.43(11)$ \\
\hline $\mathrm{C} 3-\mathrm{C} 7-\mathrm{C} 6$ & $109.04(9)$ & $\mathrm{C} 13-\mathrm{C} 12-\mathrm{C} 11$ & $110.14(10)$ \\
\hline $\mathrm{C} 3-\mathrm{C} 7-\mathrm{C} 13$ & $110.74(9)$ & $\mathrm{C} 14-\mathrm{C} 12-\mathrm{H} 12$ & $109.6(9)$ \\
\hline $\mathrm{C} 6-\mathrm{C} 7-\mathrm{C} 13$ & $108.60(8)$ & $\mathrm{C} 13-\mathrm{C} 12-\mathrm{H} 12$ & $110.0(10)$ \\
\hline
\end{tabular}




\begin{tabular}{|c|c|c|c|}
\hline $\mathrm{C} 3-\mathrm{C} 7-\mathrm{C} 8$ & $110.64(8)$ & $\mathrm{C} 11-\mathrm{C} 12-\mathrm{H} 12$ & $108.0(9)$ \\
\hline $\mathrm{C} 6-\mathrm{C} 7-\mathrm{C} 8$ & $108.67(9)$ & $\mathrm{C} 12-\mathrm{C} 13-\mathrm{C} 7$ & $109.79(10)$ \\
\hline $\mathrm{C} 13-\mathrm{C} 7-\mathrm{C} 8$ & $109.10(9)$ & $\mathrm{C} 12-\mathrm{C} 13-\mathrm{H} 13 \mathrm{~A}$ & $112.7(9)$ \\
\hline $\mathrm{C} 9-\mathrm{C} 8-\mathrm{C} 7$ & $109.55(9)$ & $\mathrm{C} 7-\mathrm{C} 13-\mathrm{H} 13 \mathrm{~A}$ & $107.3(9)$ \\
\hline $\mathrm{C} 9-\mathrm{C} 8-\mathrm{H} 8 \mathrm{~A}$ & $110.4(9)$ & $\mathrm{C} 12-\mathrm{C} 13-\mathrm{H} 13 \mathrm{~B}$ & $110.1(9)$ \\
\hline $\mathrm{C} 7-\mathrm{C} 8-\mathrm{H} 8 \mathrm{~A}$ & $108.9(8)$ & $\mathrm{C} 7-\mathrm{C} 13-\mathrm{H} 13 \mathrm{~B}$ & $108.9(9)$ \\
\hline $\mathrm{C} 9-\mathrm{C} 8-\mathrm{H} 8 \mathrm{~B}$ & $111.2(8)$ & $\mathrm{H} 13 \mathrm{~A}-\mathrm{C} 13-\mathrm{H} 13 \mathrm{~B}$ & $107.9(13)$ \\
\hline $\mathrm{C} 7-\mathrm{C} 8-\mathrm{H} 8 \mathrm{~B}$ & $110.8(8)$ & $\mathrm{C} 12-\mathrm{C} 14-\mathrm{C} 9$ & $109.43(9)$ \\
\hline $\mathrm{H} 8 \mathrm{~A}-\mathrm{C} 8-\mathrm{H} 8 \mathrm{~B}$ & $106.0(11)$ & $\mathrm{C} 12-\mathrm{C} 14-\mathrm{H} 14 \mathrm{~A}$ & $110.6(10)$ \\
\hline $\mathrm{C} 8-\mathrm{C} 9-\mathrm{C} 10$ & $110.04(10)$ & $\mathrm{C} 9-\mathrm{C} 14-\mathrm{H} 14 \mathrm{~A}$ & $109.3(9)$ \\
\hline $\mathrm{C} 8-\mathrm{C} 9-\mathrm{C} 14$ & $109.64(11)$ & $\mathrm{C} 12-\mathrm{C} 14-\mathrm{H} 14 \mathrm{~B}$ & $110.7(10)$ \\
\hline $\mathrm{C} 10-\mathrm{C} 9-\mathrm{C} 14$ & $109.70(10)$ & C9-C14-H14B & $109.7(10)$ \\
\hline $\mathrm{C} 8-\mathrm{C} 9-\mathrm{H} 9$ & $108.0(9)$ & $\mathrm{H} 14 \mathrm{~A}-\mathrm{C} 14-\mathrm{H} 14 \mathrm{~B}$ & $107.1(13)$ \\
\hline $\mathrm{C} 1-\mathrm{C} 5-\mathrm{C} 6-\mathrm{C} 7$ & $179.35(8)$ & $\mathrm{C} 11-\mathrm{C} 5-\mathrm{C} 10-\mathrm{C} 9$ & $-59.23(13)$ \\
\hline $\mathrm{C} 10-\mathrm{C} 5-\mathrm{C} 6-\mathrm{C} 7$ & $-59.25(11)$ & $\mathrm{C} 1-\mathrm{C} 5-\mathrm{C} 11-\mathrm{C} 12$ & $-178.36(10)$ \\
\hline $\mathrm{C} 11-\mathrm{C} 5-\mathrm{C} 6-\mathrm{C} 7$ & $59.41(10)$ & $\mathrm{C} 6-\mathrm{C} 5-\mathrm{C} 11-\mathrm{C} 12$ & $-59.03(12)$ \\
\hline $\mathrm{C} 5-\mathrm{C} 6-\mathrm{C} 7-\mathrm{C} 3$ & $179.79(8)$ & $\mathrm{C} 10-\mathrm{C} 5-\mathrm{C} 11-\mathrm{C} 12$ & $59.57(13)$ \\
\hline $\mathrm{C} 5-\mathrm{C} 6-\mathrm{C} 7-\mathrm{C} 13$ & $-59.44(11)$ & $\mathrm{C} 5-\mathrm{C} 11-\mathrm{C} 12-\mathrm{C} 14$ & $-60.35(13)$ \\
\hline $\mathrm{C} 5-\mathrm{C} 6-\mathrm{C} 7-\mathrm{C} 8$ & $59.12(10)$ & $\mathrm{C} 5-\mathrm{C} 11-\mathrm{C} 12-\mathrm{C} 13$ & $60.31(13)$ \\
\hline $\mathrm{C} 3-\mathrm{C} 7-\mathrm{C} 8-\mathrm{C} 9$ & $-178.86(10)$ & $\mathrm{C} 14-\mathrm{C} 12-\mathrm{C} 13-\mathrm{C} 7$ & $60.10(12)$ \\
\hline $\mathrm{C} 6-\mathrm{C} 7-\mathrm{C} 8-\mathrm{C} 9$ & $-59.18(12)$ & $\mathrm{C} 11-\mathrm{C} 12-\mathrm{C} 13-\mathrm{C} 7$ & $-60.41(12)$ \\
\hline $\mathrm{C} 13-\mathrm{C} 7-\mathrm{C} 8-\mathrm{C} 9$ & $59.07(12)$ & $\mathrm{C} 3-\mathrm{C} 7-\mathrm{C} 13-\mathrm{C} 12$ & $178.84(9)$ \\
\hline $\mathrm{C} 7-\mathrm{C} 8-\mathrm{C} 9-\mathrm{C} 10$ & $60.81(13)$ & $\mathrm{C} 6-\mathrm{C} 7-\mathrm{C} 13-\mathrm{C} 12$ & $59.14(11)$ \\
\hline $\mathrm{C} 7-\mathrm{C} 8-\mathrm{C} 9-\mathrm{C} 14$ & $-59.92(12)$ & $\mathrm{C} 8-\mathrm{C} 7-\mathrm{C} 13-\mathrm{C} 12$ & $-59.15(11)$ \\
\hline $\mathrm{C} 8-\mathrm{C} 9-\mathrm{C} 10-\mathrm{C} 5$ & $-60.73(13)$ & $\mathrm{C} 13-\mathrm{C} 12-\mathrm{C} 14-\mathrm{C} 9$ & $-60.41(14)$ \\
\hline $\mathrm{C} 14-\mathrm{C} 9-\mathrm{C} 10-\mathrm{C} 5$ & $59.97(14)$ & $\mathrm{C} 11-\mathrm{C} 12-\mathrm{C} 14-\mathrm{C} 9$ & $60.52(14)$ \\
\hline $\mathrm{C} 1-\mathrm{C} 5-\mathrm{C} 10-\mathrm{C} 9$ & $179.33(10)$ & $\mathrm{C} 8-\mathrm{C} 9-\mathrm{C} 14-\mathrm{C} 12$ & $60.44(13)$ \\
\hline $\mathrm{C} 6-\mathrm{C} 5-\mathrm{C} 10-\mathrm{C} 9$ & $59.17(13)$ & $\mathrm{C} 10-\mathrm{C} 9-\mathrm{C} 14-\mathrm{C} 12$ & $-60.51(14)$ \\
\hline
\end{tabular}

